Reproductive Toxicology

Manuscript Number: 5246R1

Title: Arterial dysgenesis and limb defects: clinical and experimental examples

Article Type: SI: Dev-Angio

Keywords: vascular transition; thalidomide; vascular disruption; human embryo; vertebrate embryo; clubfoot and vertical talus; PFFD; holt-oram syndrome

Corresponding Author: Dr. Neil Vargesson,

Corresponding Author's Institution: University of Aberdeen

First Author: Neil Vargesson

Order of Authors: Neil Vargesson; David R Hootnick

Manuscript Region of Origin: UNITED KINGDOM

Abstract: Limb malformations are amongst the most common and visible birth effects. Causes have been purported to include genetic aberrations as well as teratogens, such as thalidomide. Here we review the evidence for vascular disruption in the genesis of limb malformations through abnormal arterial transitioning and from events such as amniocentesis, uterine constriction, and through teratogen exposure. We use several clinical and experimental examples and highlight the need to understand more about the role the vascular system plays in the molecular mechanisms underpinning normal limb development. 


\begin{abstract}
Limb malformations are amongst the most common and visible birth effects. Causes have been purported to include genetic aberrations as well as teratogens, such as thalidomide. Here we review the evidence for vascular disruption in the genesis of limb malformations through abnormal arterial transitioning and from events such as amniocentesis, uterine constriction, and through teratogen exposure. We use several clinical and experimental examples and highlight the need to understand more about the role the vascular system plays in the molecular mechanisms underpinning normal limb development.
\end{abstract}




\title{
Arterial dysgenesis and limb defects: clinical and experimental examples
}

\author{
Neil Vargesson ${ }^{1, *}$ and David R. Hootnick ${ }^{2}$
}

1. School of Medicine, Medical Sciences and Nutrition, Institute of Medical Sciences. University of Aberdeen. Foresterhill. Aberdeen. AB25 2ZD. UK.

2. Departments of Orthopedic Surgery, Cellular and Developmental Biology, and Pediatrics, SUNY Upstate Medical University, 1133 Weiskotten Hall, 750 E Adams St. Syracuse. NY 13203. USA.

* Author for correspondence: n.vargesson@abdn.ac.uk; nvargesson@gmail.com

\section{Key Words:}

vascular transition, limb malformation, thalidomide, mifepristol, vascular disruption, human embryo, vertebrate embryo, clubfoot, vertical talus, PFFD (proximal femoral focal deficiency), Holt-Oram Syndrome 
Abstract

Limb malformations are amongst the most common and visible birth effects. Causes have been purported to include genetic aberrations as well as teratogens, such as thalidomide. Here we review the evidence for vascular disruption in the genesis of limb malformations through abnormal arterial transitioning and from events such as amniocentesis, uterine constriction, and through teratogen exposure. We use several clinical and experimental examples and highlight the need to understand more about the role the vascular system plays in the molecular mechanisms underpinning normal limb development.

\section{Blood vessel formation and development}

Blood vessels are essential for embryogenesis as well as for normal physiological function throughout life. In the embryo the first primitive vessels are formed by vasculogenesis, where endothelial cell precursors congregate and form vascular tubes for blood cells to pass through. This initial network is expanded upon through angiogenesis, where new vessels form through the proliferation and migration of endothelial cells and vascularize previously avascular tissues (Figure 1) [1]. Many molecules are involved in angiogenesis and one of the main triggers of angiogenesis is hypoxia which stimulates the expression of Vascular Endothelial Growth Factor to cause the proliferation and migration of endothelial cells into previously avascular tissue [1-3]. In the rapidly developing early embryo, angiogenesis occurs in the majority of tissues to ensure good oxygen and nutrient supply and the removal of waste products. Vessels can recruit a smooth muscle coating which stabilizes the vessel and also maintains blood pressure, and until the loss of the smooth muscle coat, the vessel then undergoes quiescence (Figure 1).

\section{Limb development and vascularisation of the developing limb}

In the developing human embryo, the upper limbs form around day 26 (approx. week 3.7) after fertilization followed $24 \mathrm{hrs}$ later by the lower limbs [4]. The limbs grow out from the body under the control of specialized signaling regions, specifically the Zone of Polarising Activity (ZPA) and the Apical Ectodermal Ridge (AER). These two regions in particular signal to the mesenchyme to induce mesenchymal cell proliferation and limb outgrowth as well as control differentiation of the bones and tissues of the limbs (Figure 2A) [1,5]. As the limbs grow out from the body, the multitude of tissues including muscle, tendon, nerve and bones of the limb differentiates in a proximal to distal manner, that is the humerus/femur is specified and laid down before the radius/fibula and ulna/tibia and finally the digits (Figure 2A). The vessel patterns change accordingly (Figure 2B). Vessel regression in the areas where the long bones and the future digits form occurs and this process also marks a change in the vessel pattern to the adult state (Figure 2B). By around day 56 (approx. week 8) human limbs are fully complete with fingers/toes etc.

The molecular and morphological events that underlie limb development and some limb malformations are becoming clearer and have largely been ascertained from animal models particularly the chicken and mouse (Figure 2A) [5-8]. Yet, the role of the rapidly changing vasculature in the molecular and mechanistic events underlying limb development are largely unknown.

Most of our understanding of limb vascularization has come from animal model systems and studies in human embryos/fetuses from the beginning of the $20^{\text {th }}$ century $[9,10]$. There are some 
differences in the arterial vascularization of the upper and lower limbs. Following the initiation of limb development, the main artery supplying the upper limb buds is the subclavian artery and from this a capillary network rapidly forms (via angiogenesis) throughout the outgrowing limb bud (Figure 2B) [1,3,11]. As limb development proceeds the subclavian artery name changes to become the brachial artery in the upper limb. The brachial artery itself later gives rise to the ulnar and radial arteries in the forearms. In the lower limbs, arterial vascularization also proceeds through angiogenesis but is a little more complicated. Initially primitive arteries from the umbilical artery traverse the forming limb bud as the axial and external iliac arteries. The external iliac artery eventually becomes the femoral artery, supplying the upper leg. The axial artery traverses the outgrowing leg bud to supply the distal regions of the leg bud and further divides into the fibular (peroneal) arteries (Figure 3). As the leg bud develops, there is further splitting of the femoral artery to produce further arterial supply to the forming bones of the limbs (Figure 3).

In addition, there is also vessel regression as well as formation of composite arteries. The fibular (peroneal artery), which primarily provides the blood supply to the fibula, is one such composite artery; it contains four elements/segments of three earlier embryonic arteries, which have undergone repeated rounds of angiogenesis and stabilization to achieve the adult configuration (Figure 3) $[9,10,12]$.

98

\section{Embryonic to adult arterial transition}

100

101

102

103

104

105

106

107

108

Elegant analyses in human embryos have demonstrated that, in early limb buds, a capillary network is evident. As development proceeds, the vascular pattern differentiates into defined vessels initially in the proximal tissue and then finally in the distal tissue, where the digits form last (Figure 3) [9, 10, 13-16]. Approximately five weeks after fertilization, single axial arteries of both the upper and lower extremities begin the transition to more complex arterial patterns (Figure 3). The arterial transitions coincide with the long bone transitions from mesenchymal primordia (anlage) to chondral and then osseous structures. Arterial alterations occur prior to and during the organization of the muscles, tendons and nerves but after specification/patterning of the limb is completed [17]. The upper and lower human limb embryonic arterial transitions have been completed prior to the $8^{\text {th }}$ week of development, resulting in the adult arterial pattern (Figure 3) $[9,1013,14,18]$.

In the chicken embryo the transition of the arterial pattern of the limbs from embryonic to mature state has occurred by approximately day 8 of development. As in the the human embryo, the arterial transitions occur during and after the specification and patterning of the bony elements has occurred. The arterial transition likely allows the bones of the limb to form correctly and in the correct positions [11, 19]. Much of our understanding of the processes of limb vascularization has come from studying chicken limb development [1, 2, 11, 19]. Unlike vessels in other regions of the chicken embryo, the early developing limb vascular network remains devoid of vascular smooth muscle cells, which lend protection and support to the vessels. Not until relatively late in limb development, after the adult vascular pattern has started to become established are vascular smooth muscle cells seen upon limb vessels (Figure 2B) [11, 20]. Indeed, the brachial artery starts to recruit smooth muscle first, in chicken embryos from around HH St25 (E5; about 2.5 days after limb development has started, which is relatively late in development as the limbs buds are well advanced in outgrowth) (Figure 2A). Other vessels only start recruiting smooth muscle much later in development [11, 20]. By comparison, vessels in 
other regions of the embryo, including the head, contain vascular smooth muscle coats by these stages [1, 20, 21]. Smooth muscle protects the vessel and prevents angiogenesis. In order for angiogenesis to re-occur, the smooth muscle coat needs to be lost [1]. Since the developing limbs are rapidly growing and changing, the developing limb requires a vasculature that adapts to the changing environment quickly; thus, a smooth muscle coat would be an obstacle to early development. Absence of the smooth muscle coat presumably permits rapid changes in limb outgrowth to occur. However, this also makes the vessels more susceptible to potential injury and damage $[1,20]$.

\section{Embryonic to adult arterial transition failure and limb defects}

Whilst there may be a variation of limb arterial branching patterns in up to $20 \%$ of normal adult humans [13], a failure of correct vessel patterning or a failure of the embryo to adult vascular transition has been associated with limb defects in humans [12, 22-27] and in animal models [20, 28-31].

Indeed, through radiographic and pathologic analyses of dysmorphic human lower limbs, the combined associations of reduction or absence of the proximal femur [22, 32], the fibula [33] and midline metatarsals [23] suggests the possibility of a temporal and anatomic linkage of the skeletal changes to failure of embryonic arterial transition, when the long bones are first forming $[9,10,12,15,18]$. Arteriography of a series of such affected limbs further revealed abnormal arterial patterns, which have demonstrated persistence of primitive embryologic arteries, absence of one of the normal adult arteries and failure of formation of the plantar arch of the foot [12, 32$35]$.

For example, some cases of congenital fibular deficiency (Figure 4A-C) [12] and radial aplasia [36] have been described indicating the vascular pattern of the limbs appears to be intermediate between an embryonic and adult state, suggesting the long bones might not form or might form incorrectly due to the misplacement or mis-positioning of the vessels. Furthermore, the foot is typically supplied with three arteries, the posterior tibial, anterior tibial and the fibular (peroneal) (Figure 3B). In the debilitating condition clubfoot, where the foot points downward, toes are turned inward and the bottom of the foot, that would normally be walked on is twisted inward, the anterior tibial artery is most consistently reduced in length in the most severely deformed limbs and absent in the distal foot. The lack of alternative arterial pathways has been proposed to explain why the foot 'clubs' in such cases (Figure 4D) [17, 24, 35, 37, 38]. In contrast, in cases of the congenital vertical talus deformity, where the foot is pointed upwards, rigidly, causing the toes to point up and out with loss of the arch of the foot, the posterior tibial artery is most consistently significantly reduced or sometimes missing [24, 34, 35, 38]. Similarly, arteriography of the limbs of children with proximal femoral focal deficiency (PFFD; congenitally short limb), where the femur may be significantly reduced in length and can vary morphologically from just a distal remnant of the femur to an almost normal sized femur, has revealed distinctly consistent arterial changes in a majority of the affected limbs [32]. The changes may include reduction of the diameter and length of the femoral arteries and/or preservation of the primitive sciatic (ischiadic) artery [32].

An abnormal arterial pattern in the developing limb could put the limb at risk for subsequent malformation as, for example, there may be a reduced potential for collateral vascular circulation 
resulting in tissue damage or loss. Conditions that antagonise blood flow through the remaining arteries could then lead to tissue damage. This tissue damage could, in turn, interfere with the developmental specification or differentiation of limb structures, leading to bony and/or soft tissue abnormalities [12, 17, 24, 26, 33, 37].

\section{Timing of arterial dysgenesis}

Limb development and arterial transition occur between 5 and 8 weeks of human embryonic development. This coincides with several periods of very rapid growth, considering that the crown rump length of $10 \mathrm{~mm}$ at $5 \frac{1}{2}$ weeks grows to $20 \mathrm{~mm}$ at 7 weeks, thus effectively doubling in length over a ten day period. The next most significant period of growth occurs between 7 and 8 weeks, over a seven day period, when the embryo enlarges from a crown rump length of 20 $\mathrm{mm}$ to a crown rump length of $30 \mathrm{~mm}$. That is a 50\% enlargement over a seven day period [4, 39]. During these times, the outgrowth of the upper and lower extremities is rapidly progressing, after the specification of the limbs has begun and while the embryonic arterial alterations are occurring [4, 12, 17, 37, 39]. Since the critical period for the initiation of bone development, beginning as mesenchymal condensations, occurs subsequent to the fifth week of embryonic life, coinciding with the time that the adult arterial pattern of vessels is emerging, it is possible that both the arterial and skeletal systems simultaneously are mutually vulnerable to teratogenic insults. Thus, the maldevelopment of the bones and the embryonic arterial pattern may be intimately related $[23,33]$. The evidence of embryonic arterial dysgenesis in association with a variety of skeletal malformations and deletions has provided an explanation for the timing, pathogenesis and distribution of some limb malformations.

Indeed, comparisons of the estimates of the upper femoral vs. lower leg dysmorphologies suggest that the estimated timings of the respective arterial embryonic dysgeneses are associated with the skeletal dysmorphologies observed. These findings are consistent with a progressively more distal model of arterial development, after the completion of limb specification [9, 10, 12, $17,18,23,32,37]$. Indeed, the critical time for connection between the femoral artery and the sciatic (ischiadic) artery in the femoral region of the lower extremity has been estimated to occur between the 12 and $14 \mathrm{~mm}$ stages of embryonic development (approximately week 6) (Figure 3B) $[9,10,32]$; the critical time for arterial changes of the more distal part of the lower extremity in the tibial and fibular region has been estimated to occur between the 19 and $22 \mathrm{~mm}$ stages of embryonic development (approximately week 7) (Figure 3B) [12, 23]. Hence many limb malformations may be interpreted as post-specification events, which occur after the bony elements have been specified but which are then damaged, diminished or radiologically absent through failure or loss of appropriate vascularization resulting in smaller or missing bones. More elementally, such careful dissections and/or detailed imaging of human malformed limbs has revealed that such limb malformations possess an arterial pattern typical of embryonic stages and/or missing or misplaced vessels consistent with a failure of correct vascularization of the involved bone $[12,17,22,23,32,33,34,35,37]$. Failure of arteries to develop to vascularize the condensing cartilage precursor of the long bones will have intuitively resulted in the loss or reduction in the length of the long bone (Figure 4). For example, late arterial invasion of the tibia has been linked to the shortening and bending of the bone due to a delayed formation of the perichrondral ring, which encases the diaphysis (growth plate) of the long bones [33, 40]. Likewise, in limbs with PFFD, a significantly reduced arterial supply to the femur has been identified that is linked to femoral shortening [32]. Further, the distal $2 / 3$ of the fibula is supplied 
by the fibular (peroneal) artery, whilst the proximal $1 / 3$ is supplied by the anterior tibial artery; thus, loss of either one of these vessels during fibula differentiation might result in fibula deficiency or diminution $[9,10,12]$. Finally, in the upper limb, loss of the radial artery has been purportedly linked to the underlying cause of radial aplasia (loss of the radius) [36].

The failure of arteries to vascularize their respective bony precursors could explain many limb abnormalities including long bone loss, long bone bending as well as changes to digital patterns due to the loss of or shortening of the long bones. Given the arterial transition occurs as the bones have been specified and are starting to condense, the damage is thus, post-specification.

What could cause the blood vessels to not form correctly, or fail to transit to the adult state or simply mispattern to cause limb anomalies?

\section{Molecular changes resulting in vascular disruption}

We know there are many human (and animal) limb defects associated with genetic mutations and signaling changes $[6,7]$; perhaps these are caused in early development before specification of the bony elements or perhaps the resulting signaling changes alters vascularization of the developing limbs and the arterial transition and branching events?_For example, in some patients with Holt-Oram Syndrome, a condition caused by a mutation in the gene Tbx5, upper limb reduction ranging in severity from loss of the thumb to phocomelia is seen [41]. A reduced peripheral vasculature in the affected limb/s has been reported in some patients [42]. Whether this is secondary to the limb malformation or could actually be responsible is unclear.

Furthermore, recent work in animal models does suggest that failure of the forming bones to receive the correct vascularization at the right time does result in bone outgrowth failure. For example, a study where the neurotrophic factor, TrkA, essential to direct innervation and promote vascularization of the outgrowing bone, when knocked out in mouse embryos result in smaller bones in the limb due to a failure of vascularization of the bone [43]. Moreover, molecular signals essential for blood vessel formation and identity, such as the Ephrins, members of the Notch signaling pathway and members of the VEGF signaling pathway, are also essential for proper bone vascularisation [44, 45]. Thus, if molecular signals fail to induce correct vascularization of the forming bones, those bones do not form correctly.

\section{Trauma during pregnancy leading to arterial dysgenesis or vascular disruption -Occlusion of vessels}

Pregnancy significantly increases the chances of thrombotic events, frequently due to changes in blood coagulation whilst pregnancy is ongoing to prevent excess blood loss during pregnancy and childbirth [46]. However, this could also increase the chance of thrombotic events in the embryo. Some studies have proposed that vessel occlusion in the embryo through thrombi through the placenta or from the heart or injured tissue could result in tissue damage leading to malformation in humans $[25,27,47,48]$ and in animals [29, 31, 49].

Indeed, one study in chicken embryos demonstrated that following occlusion of the carotid arteries severe brain anomalies such as anencephaly could be induced [49]. The study simply demonstrates that the carotid arteries were missing and tissue distal to the occlusion never formed [49]. This is an example of downstream tissue loss after vascular obstruction. 


\section{-Hemorrhage}

Hemorrhage early in embryogenesis has also been linked in the genesis of malformations [5052]. Indeed localized hematomas have been observed in spontaneously aborted human fetuses, without determination that the hematoma was the cause of the fetal death [53]. In addition focal hematomas or hemangiomas have been observed on the forehead of thalidomide damaged babies $[51,54,55]$. Hemorrhage induction in rat embryos through injection of drugs like aspirin [56] or through amniocentesis [57] has been shown to result in limb defects in rat fetuses, where increased uterine contractions in a smaller uterine space are purported to have caused damage, specifically via hemorrhaging, in the forming hand and footplates of the embryos; this can result in constriction bands, loss of digits and acrosyndactyly [57]. Similarly, in chicken embryos, after vessels were physically injured resulting in hemorrhage, embryonic development was impaired [52]. The hemorrhage most likely initiated a failure of differentiation of mesoderm and interfered with signaling processes, resulting in such dysmorphologies.

These events can result from external influences such as drug exposure (see next section) or through trauma, even from procedures that are seemingly simple. As, for example, amniocentesis, a method of analysis of the chromosome karyotype of the embryo/fetus involves aspirating amniotic fluid. It can lead to uterine constriction due to the reduction in amniotic fluid volume, resulting in damage to the limbs. Moreover, chorionic villus sampling, also used as a prenatal diagnostic tool, has been linked to terminal transverse limb malformations through disruptive vascular events, through hemorrhaging or cell death/tissue loss and/or hemangioma formation [58]. Although these events rarely occur, and most procedures occur without untoward consequences, these studies do highlight the need to monitor pregnancies without reduction of amniotic fluid to minimise damage to the extremities if uterine constriction does occur. In addition, placental damage itself from vascular insufficiency or from trauma to the uterus could result in thrombi that occlude vessels or cause hemorraging in the placenta restricting blood flow to the embryo potentially resulting in growth retardation of embryonic tissues [58].

\section{Drugs taken during pregnancy leading to vascular disruption \\ -Thalidomide}

Of course, environmental influences can also cause vascular anomalies in embryos, these include exposure to drugs like phenytoin, cocaine and valproate [58-60]. Perhaps the most infamous are thalidomide and more recently, misoprostol.

Thalidomide has been shown to be antiangiogenic in rabbit and rodent cornea assays [61, 62] and in chicken and zebrafish embryos [21, 63-66]. Indeed, the drugs antiangiogenic action is widely viewed as part of the key in the drugs teratogenic actions where the drug may target angiogenic, smooth muscle negative vessels resulting in their loss, or preventing them to transition to an adult state as well as then resulting in changes in molecular signaling pathways and ultimately tissue loss/malformation [67-69].

It is of further interest to note that several studies in thalidomide survivors (children and adult) with limb anomalies, have reported cardiovascular malformations and vascular changes and/or missing vessels in the limbs which might help explain the damage [70, 71]. 
The hypothesis is that thalidomide induces vessel loss or may interfere with the vascular transition which could lead to localized cell death resulting in missing or lost tissue, resulting in bony elements being shorter or lost [67, 68]. As the time sensitive window of thalidomide embryopathy is day 20-36 after fertilization (day 34-50 after the last menstrual cycle), when vascular transition of the limbs is occurring and when proximal bony elements are condensing and starting to differentiate (Figure 2A), inhibition of vascularization could well result in the deficiency of or shortening of the forming long bones which could vary depending on the arterial alterations occurring at that time $[67,68]$.

\section{-Misoprostol}

Misoprostol is a synthetic analog of Prostaglandin E1 and used to prevent gastric side effects caused by nonsteroidal anti-inflammatory drugs. It also causes endometrial bleeding and uterine contractions and so has been used as an abortificient, particularly in Brazil [58, 72]. Sadly, it has been shown to cause birth defects in children when the drug was used improperly [58]. The injuries observed in such affected infants appears similar to the damage seen in thalidomide damaged children [73] with limbs, facial and internal organs all affected. Moreover, the drug is thought to cause vascular disruption in tissues likely due to increased uterine contractions that Misoprostol induces (as part of the abortifacient process) resulting in hypoperfusion of the embryo/fetus and blood entrapment/hemorrhaging [58, 74, 75].

Several other agents, including Valproic acid, can also cause vascular anomalies during embryonic development. In chicken embryos valproate exposure produces a range of defects similar to those in humans including neural and cardiac defects [76] and limb reduction defects [77]. Furthermore, recent work demonstrated that popular anti-cancer antiangiogenic agents, including Sunitinib, Sorafenib, Everolimus are also teratogenic and can cause a range of defects including limb anomalies [78].

\section{Conclusion}

Blood vessels in the developing embryonic limbs change rapidly to accommodate the outgrowth of the limbs and the differentiation processes needed to allow the complex combinations of tissues to form. The morphology of the bony pattern of the limbs evolves as vascular pattern is transforming to a mature, adult-like state. Noxious events which prevent the proper arterial transitioning, can prevent vessels from correctly invading the forming precursors of the bones resulting in a variety of defects. Similarly, injury resulting from hemorrhage, uterine constriction, physical damage, drug exposure, molecular signalling failure or interference could also result in such vascular changes. Such loss or inhibition of vessels or disruption of blood flow results in poorly perfused tissues and bones leading to deficiency and/or malformation. As arterial transition and bone formation are occurring at similar time points in embryonic development, such vascular damage could explain a variety of limb anomalies.

Our understanding of the normal mechanisms underlying and controlling angiogenesis and how the developing vasculature influences normal limb development, particularly the underlying molecular signaling events, remains unclear. Indeed, recent models of pattern formation in limb development have seemed solely focused on molecular mechanisms $[8,79,80]$ yet largely ignore 
the developing vasculature of the limbs which clearly fulfills an essential role in limb development.

\section{Acknowledgements}

This article is dedicated to Dr David S. Packard Jr. With thanks to Dr John DeSesso, Dr Lewis B. vascular disruption, particularly arterial dysgenesis and limb defects.

This research did not receive any specific grant from funding agencies in the public, commercial, or not-for-profit sectors.

\section{References}

1. N. Vargesson, Vascularisation of the developing chick limb bud: role of the TGFbeta signaling pathway. J Anat. 202 (2003) 93-103.

2. A.I. Caplan, The vasculature and limb development. Cell Differ. 16 (1985) 1-11.

3. W. Risau, Mechanisms of angiogenesis. Nature 386 (1997) 671-674.

4. R. O'Rahilly, E. Gardner, The timing and sequence of events in the development of the limbs in the human embryo. Anat Embryol. 148 (1975) 1-23.

5. C. Tickle, How the embryo makes a limb: determination, polarity and identity. J Anat. 227 (2015) 418-30.

6. R. Zeller, The temporal dynamics of vertebrate limb development, teratogenesis and evolution. Curr Opin Genet Dev. 20 (2010) 384-390.

7. A.Zuniga, R. Zeller, S. Probst, The molecular basis of human congenital limb malformations. Wiley Interdiscip Rev Dev Biol. 1 (2012) 803-822.

8. I. Delgado, M. Torres, Gradients, waves and timers, an overview of limb patterning models. Semin Cell Dev Biol. 49 (2016) 109-115.

9. H.D. Senior, The development of the arteries of the human lower extremity. Am J Anat. 25 (1919) 55-95.

10. H.D. Senior, An interpretation of the recorded arterial anomalies of the human leg and foot. J Anat. 53 (1919) 130-171.

11. R.F Drushel, D.G. Pechak, A.I. Caplan, The anatomy, ultrastructure and fluid dynamics of the developing vasculature of the embryonic chick wing bud. Cell Differ. 16 (1985) 13-28.

12. E. M. Levinsohn, D. R. Hootnick, D. S. Packard Jr, Consistent arterial abnormalities associated with a variety of congenital malformations of the human lower limb. Invest Radiol. 26 (1991) 364-373. 
13. M. Rodriguez-Niedenfuhr, G. J. Burton, J. Deu, J. R. Sanudo, Development of the arterial pattern in the upper limb of staged human embryos: normal development and anatomic variations. J. Anat. 199 (2001) 407-417.

14. M. Rodriguez-Niedenfuhr, T. Vazquez, I. G. Parkin, J. R. Sanudo, Arterial patterns of the human upper limb: update of anatomical variations and embryological development. Eur J Anat. 7 (2003) Suppl. 1:21-28.

15. E. Singer, Embryological pattern persisting in the arteries of the arm. Anat Rec. 55 (1933) 403-409.

16. H. D. Senior, A note on the development of the radial artery. Anat Rec. 32 (1926) 220-221.

17. D. S. Packard Jr, E. M. Levinsohn, D. R. Hootnick, Most human lower limb malformations appear to result from postspecification insults. Prog Clin Biol Res. 383A (1993) 417-426.

18. H. D. Senior, Abnormal branching of the human popliteal artery. Am J Anat. 44 (1929) 111120.

19. E. M. Levinsohn, D. S. Packard, E. M. West, D. R. Hootnick, Arterial anatomy of chicken embryo and hatchling. Am J Anat. 169 (1984) 377-405.

20. N. Vargesson, E. Laufer, Smad7 misexpression during embryonic angiogenesis causes vascular dilation and malformations independently of vascular smooth muscle cell function. Dev Biol. 240 (2001) 499-516.

21. C. Therapontos, L. Erskine, E. R. Gardner, W. D. Figg, N. Vargesson, Thalidomide induces limb defects by preventing angiogenic outgrowth during early limb formation. Proc Natl Acad Sci U S A. 106 (2009) 8573-8578.

22. C. Hamanishi, Congenital short femur. Clinical, genetic and epidemiological comparison of the naturally occurring condition with that caused by thalidomide. J Bone Joint Surg Br. 62 (1980) 307-320.

23. D. R. Hootnick, E. M. Levinsohn, D. S. Packard Jr, Midline metatarsal dysplasia associated with absent fibula. Clin Orthop. 150 (1980) 203-206.

24. D. R. Hootnick, D. S. Packard Jr, E. M. Levinsohn, A. Wladis, A vascular hypothesis for the etiology of Clubfoot, in G. W. Simons (Eds), The Clubfoot: The Present and a View of the future. Springer-Verlag, New York. 1994. pp. 48-58.

25. H. E. Hoyme, K. L. Jones, M. I. Van Allen, B. Saunders, K. Benirschke, Vascular pathogenesis of transverse limb reduction defects. J Pediatr 101 (1982) 839-843.

26. D. S. Packard Jr, E. M. Levinsohn, D. R. Hootnick, Teratological implications of soft tissue abnormalities found in human lower limbs with bony defect, in R. N. Feinberg, G. K. Sherer, R. 
Auerbach (Eds), The Development of the Vascular System. Karger, Basel. Issues in Biomedicine 14 (1991) 157-169.

27. M. I. Van Allen, Fetal vascular disruptions: mechanisms and some resulting birth defects. Pediatr Ann. 10 (1981) 219-233.

28. H. J. Bagg, Hereditary abnormalities of the limbs, their origin and transmission. II. A morphological study with special reference to the etiology of clubfeet, syndactylism, hypodactylism and congenital amputation in the descendants of x-rayed mice. Am J Anat. 43 (1929) 167.

29. H. S. N. Greene, J. A. Saxton, Hereditary bracydactylia and allied abnormalities in the rabbit. J Exp Med. 69 (1939) 301-319.

30. L. A. Kennedy, T. V. Persaud, Pathogenesis of developmental defects induced in the rat by amniotic sac puncture. Acta Anat (Basel). 97 (1977) 23-35.

31. C. Petter, J. Bourbon, J-P. Maltier, A. Jost, Simultaneous prevention of blood abnormalities and hereditary congenital amputations in a Brachydactylous rabbit stock. Teratology 15 (1976) $149-158$.

32. J. Chomiak, M. Horak, M. Masek, M. Frydrychova, P. Dungl P, Computed Tomographic Angiography in Proximal Femoral Focal Deficiency. J Bone Joint Surg Am. 91 (2009) 19541964.

33. D. R. Hootnick, E. M. Levinsohn, P. A. Randall, D. S. Packard, Vascular dysgenesis associated with skeletal dysplasia of the lower limb. J. Bone Joint Surg. 62-A (1980) 1123-1129.

34. D. R. Hootnick, D. S. Packard Jr, E. M. Levinsohn, M. R. Lebowitz, J. P. Lubicky, The anatomy of a congenitally short limb with clubfoot and ectrodactyly. Teratology 29 (1984) 155164.

35. L. Kruse, C. A. Gurnett, D. Hootnick, M. B. Dobbs, Magnetic resonance angiography in clubfoot and vertical talus. Clin Orthop Relat Res. 467 (2009) 1250-1255.

36. M. I. Van Allen, H. E. Hoyme, K. L. Jones, Vascular pathogenesis of limb defects. I. Radial artery anatomy in radial aplasia. J Pediatr 101 (1982) 832-838.

37. D. S. Packard Jr, E. M. Levinsohn, D. R. Hootnick, Extent of duplication in lower-limb malformations suggests the time of the teratogenic insult. Pediatrics 91 (1993) 411-413.

38. H. Sodre, S. Bruschini, L. A. Mestriner, F. Miranda Jr, E. M. Levinsohn, D. S. Packard Jr, R. J. Crider Jr, R. Schwartz, D. R. Hootnick, Arterail abnormalities in Talipes Equinovarus as assessed by angiography and the Doppler technique. J Pediatr Orthop. 10 (1990) 101-104. 
39. R. O'Rahilly, F Muller, Developmental stages in human embryos, in Developmental stages in human embryos. Carnegie Institution of Washington. Publication 637. 1987. pp.1-263.

40. H. J. Merker, Considerations on the problem of critical period during the development of the limb skeleton. Birth Defects 13 (1977) 179-202.

474 41. C. A. Goldfarb, L. B. Wall, Holt-Oram Syndrome. J Hand Surg. 39 (2014) 1646-1648.

42. C. T. DuPre, R. M. Fincher, Holt-Oram syndrome associated with hypoplastic peripheral vasculature and midsystolic click. South Med J. 86 (1993) 453-456.

43. R. E. Tomlinson, Z. Li, Q. Zhang, B. C. Goh, Z. Li, D. L. J. Thorek, L. Rajbhandari, T. M. Brushart, L. Minichiello, F. Zhou, A. Venkatesan, T. L. Clemens, NGF-TrkAsignaling by sensory nerves coordinates the vascularization and ossification of developing endochrondral bone. Cell Rep. 16 (2016) 2723-2735. Doi:10.1016/j.celrep.2016.08.002 of hematopiesis and osteogenesis by blood vessel-derived signals. Ann Rev Cell and Dev Biol 32 (2016) In Press. DOI: 10.1146/annurev-cellbio-111315-124936

45. T. Hashimoto, M. Tsuneki, T. R. Foster, J. M. Sanatan, H. Bai, M. Wang, H. Hu, J. J. Hanisch, A. Dardik, Membrane-mediated regulation of vascular identity. Birth Defects Res C Embry Today 108 (2016) 65-84.

46. A. H. James, Thrombosis in pregnancy and maternal outcomes. Birth Defects Research (Part C) 105 (2015) 159-166.

47. C. F. Parsa, M. P. Robert, Thromboembolism and congenital malformations. JAMA Opthalmol. 131 (2013) 439-447.

491 48. M. I. Van Allen, J. C. Jackson, R. H. Knopp, R. Cone, In utero thrombosis and neonatal 492 gangrene in an infant of a diabetic mother. Am J Med Genet. 33 (1989) 323-327.

493 49. F. S. Vogel, J. L. McClenahan, Anomalies of major cerebral arteries associated with 494 congenital malformations of the brain. Am J Pathol. 28 (1952) 701-723.

495 50. D. Poswillo, The pathogenesis of the first and second branchial arch syndrome. Oral Surg 496 Oral Med Oral Pathol. 35 (1973) 302-328.

497 51. D. Poswillo, Hemorrhage in development of the face. Birth Defects Orig Artic Ser. 11 (1975) 498 61-81.

499 52. R. B. Stark, H. Washio, M. Deforest, S. Broque, The role of vascular deficiency in the 500 production of congenital malformation; preliminary report. Plast Reconstr Surg. 35 (1965) 478501532. 
53. C. T. Grabowski, Embryonic oxygen deficiency - a physiological approach to analysis of teratological mechanisms. Adv Teratol. 4 (1970) 154.

54. W. Lenz, K. Knapp, Foetal malformations due to thalidomide. German Med Monthly 7 (1962) 253-258.

55. R. W. Smithells, C. G. Newman, Recognition of thalidomide defects. J Med Genet. 29 (1992) 716-723.

56. D. E. Poswillo, D. Sopher D, Malformation and deformation in the animal embryo. Teratology 4 (1971) 498.

57. Y. Kino, Clinical and experimental studies of the congenital constriction band syndrome, with an emphasis on it etiology. J Bone Joint Surg Am. 57 (1975) 636-643.

58. L. B. Holmes, Teratogen-induced limb defects. Am J Med Genet. 112 (2002) 297-303.

59. P. A. Dennery, Effects of oxidative stress on embryonic development. Birth Defects Res C 81 (2007) 155-162.

60. M. Cassina, G. A. Cagnoli, D. Zuccarello, E. Di Gianantonio, M. Clementi, Human teratogens and genetic phenocopies. Understanding pathogenesis through human genes mutation. Eur J Med Genet (2016) In Press. DOI: 10.1016/j.ejmg.2016.09.011

61. R. J. D'Amato, M. S. Loughnan, E. Flynn, J. Folkman, Thalidomide is an inhibitor of angiogenesis. Proc Natl Acad Sci U S A. 91 (1994) 4082-4085.

62. B. M. Kenyon, F. Browne, R. J. D'Amato. Effects of thalidomide and related metabolites in a mouse corneal model of neovascularisation. Exp Eye Res. 64 (1997) 971-978.

63. A. Jurand, Early changes in limb buds of chick embryos after thalidomide treatment. J Embryol Exp Morphol. 16 (1966) 289-300.

64. C. Mahony, L. Erskine, J. Niven, N. H. Greig, W. D. Figg, N. Vargesson, Pomalidomide is nonteratogenic in chicken and zebrafish embryos and nonneurotoxic in vitro. Proc Natl Acad Sci U S A. 110 (2013) 12703-12708.

65. K. P. Tamilarasan, G. K. Kolluru, M. Rajaram, M. Indhumathy, R. Saranya, S. Chatterjee, Thalidomide attenuates nitric oxide mediated angiogenesis by blocking migration of endothelial cells. BMC Cell Biol. 7 (2006) 17

66. T. Yabu, H. Tomimoto, Y. Taguchi, S. Yamaoka, Y. Igarashi, T. Okazaki, Thalidomideinduced antiangiogenic action is mediated by ceramide through depletion of VEGF receptors, and is antagonized by sphingosine-1-phosphate. Blood 106 (2005) 125-134. 
67. N. Vargesson, Thalidomide-induced limb defects: resolving a 50-year old puzzle. BioEssays 31 (2009) 1327-1336.

68. N. Vargesson, Thalidomide-induced teratogenesis: history and mechanisms. Birth Defects Res C Embryo Today. 105 (2015) 140-156.

69. N. Vargesson, Thalidomide embryopathy: an enigmatic challenge. ISRN Developmental Biology Vol 2013 (2013) Article ID 241016. http://dx.doi.org/10.1155/2013/241016

70. W. A. Maier, Thalidomide embryopathy and limb defects: experiences in habilitation of children with ectromelias. Arch. Dis. Childh. 40 (1965) 154.

71. T. Tajima, T. Wada, A. Yoshizawa, T. Masuda, T. Okafuji, T. Nakayama, K. Hasuo, Internal anomalies in thalidomide embryopathy: results of imaging screening by CT and MRI. Clin Radiol. 71 (2016) 1199.e1-7 doi: 10.1016/j.crad.2016.07.015

\section{C. H. Gonzalez, M. J. Marques-Dias, C. A. Kim, S. M. Sugayama, J. A. Da Paz, S. M.} Huson, L. B. Holmes, Congenital abnormalities in Brazilian children associated with misoprostol misuse in first trimester pregnancy. Lancet. 351 (1998) 1624-1627.

73. M. T. Miller, L. Ventura, K. Stromland, Thalidomide and misoprostol: ophthalmologic manifestations and associations both expected and unexpected. Birth Defects Res A Clin Mol Teratol. 85 (2009) 667-676.

74. T. da Silva Dal Pizzol, F. P. Knop, S. S. Mengue, Prenatal exposure to misoprostol and congenital anomalies: systematic review and meta-analysis. Reprod Toxicol. 22 (2006) 666-671.

75. F. R. Vargas, L. Schuler-Faccini, D. Brunoni, V. F. Meloni, S. M. Sugayama, L. Albano, J. C Jr. Llerena, J. C. Almeida, A. Duarte, D. P. Cavalcanti, E. Goloni-Bertollo, A. Conte, G. Koren, A. Addis, Prenatal exposure to misoprostol and vascular disruption defects: a case control study. Am J Med Genet. 95 (2000) 302-306.

76. P. G. Kelly, C. M. Regan. Studies on valproate-induced perturbations of neurulation in the explanted chick embryo. Toxicology 71 (1992) 137-144.

77. A. I. Whitsel, C. B. Johnson, C. J. Forehand. An in ovo chicken model to study the systemic and localized teratogenic effects of valproic acid. Teratology 66 (2002) 153-163.

78. S. L. Beedie, C. Mahony, H. M. Walker, C. H. Chau, W. D. Figg, N. Vargesson, Shared mechanism of teratogenicity of anti-angiogenic drugs identified in the chicken embryo model. Scientific Reports 6:30038 (2016) DOI: 10.1038/srep30038.

79. C. Tabin, L. Wolpert L, Rethinking the proximodistal axis of the vertebrate limb in the molecular era. Genes Dev. 21 (2007) 1433-1442. 
566 80. A. Zuniga, Next generation limb development and evolution: old questions, new

567 perspectives. Development 142 (2015) 3810-3820.

568 81. D.R. Hootnick, D. S. Packard Jr. E. M. Levinsohn, R. Constantine, Postoperative necrosis in 569 Clubfoot: recent findings and review. Orthopedics International Edition 1 (1992) 48-68. 


\section{$573 \quad$ Figure Legends}

\section{$574 \quad$ Figure 1}

\section{Vasculogenesis and Angiogenesis}

576 Endothelial cell precursors coalesce to form a clump of cells which then form a lumen to form

577 the primitive vessels in a process termed vasculogenesis - this is the de novo formation of the

578 first vessels. The vessels undergo angiogenesis following signal stimulation which result in

579 endothelial cell proliferation and migration. Once vessels have reached their target they can

580 recruit smooth muscle cells to become stable and to mature. In order to undergo further

581 angiogenesis the vascular smooth muscle coat needs to be shed. Some of the signalling

582 molecules involved in angiogenesis and vessel maintenance are detailed.

583 Figure reproduced with permission from N. Vargesson, Vascularisation of the developing chick

584 limb bud: role of the TGFbeta signalling pathway. J. Anat. 202 (2003) 93-103. [1]

\section{$585 \quad$ Figure 2}

\section{Limb development and limb vascularisation}

587 A.Generalised schematic of limb development. In the early limb bud Fgf8 is released from the 588 the apical ectodermal ridge (AER) and signals to the zone of polarising activity (ZPA) to produce Shh which then establishes and maintains a feedback loop to allow mesenchymal cell proliferation and limb bud outgrowth. As limb development proceeds differentiation of the bones of the limb starts in a proximal to distal manner with the humerus forming first. The AER-ZPA feedback loop is maintained and induces many other signals involved in limb formation and patterning including Hox genes which also play a role in patterning the different bony elements.

B.Vascularisation of the developing chicken embryo forelimb. Upper panels are ink injected limb buds at different stages of development to highlight the forming and rapidly changing vasculature. The numbers refer to the stage of development of the embryo: $18=$ day $2.5 ; 23=$ day $4 ; 26=$ day $5 ; 30=$ day 7 . The limb bud grows out rapidly and the vasculture changes rapidly to accommodate the outgrowth. Arrows demarcate the avascular zone between the angiogenic vessels and the limb tip. Bottom panel are confocal microscope images of limbs at the equivalent stages as the corresponding upper panel showing an antibody stain for vascular smooth muscle actin. This highlights that the subclavian artery is the first and only artery to recruit smooth muscle at around day 5 of development and by day 7 of development, the whole of the subclavian artery and the proximal parts of the radial and ulnar arteries have smooth muscle but very few other vessels are smooth muscle positive at this point. The recruitment of smooth muscle to the subclavian artery coincides with the visualisation of the humerus proximally. The recruitment of smooth muscle to the radial and ulnar arteries coincides with the

608 appearance of the radius and ulna condensations. Vascular smooth muscle negative vessels are more angiogenic and permit rapid change to accommodate the outgrowing limb bud, however 
they are also weaker and more susceptible to teratogenic insults. The black asterisk in the handplate denotes vessel regression in the future areas where the digits will condense and form.

611 Figure A reproduced with modification from N. Vargesson, Thalidomide-induced teratogenesis:

612 history and mechanisms. Birth Defects Res C Embryo Today. 105 (2015) 140-156. [68]

613 Figure B reproduced with permission from N. Vargesson, Vascularisation of the developing

614 chick limb bud: role of the TGFbeta signalling pathway. J. Anat. 202 (2003) 93-103. [1]

\section{Figure 3}

\section{Embryo to adult arterial transition}

618 A Upper limb arterial transition. The vessel capillary plexus network seen in the very early limb 619 bud around Stage 12 (30 days) disappears and differentiates into the adult pattern following the 620 appearance of the skeletal elements.

621 Stage's refer to the Carnegie staging system of human embryo development (O'Rahilly and 622 Muller, 1987). Stage 12, 30 days (approx. 4.3 weeks); Stage 13, 32 days (approx. 4.6 weeks); 623 Stage 14, 33 days (approx. 4.7 weeks); Stage 15, 36 days (approx. 5.1 weeks); Stage 17, 41 days 624 (approx. 5.9 weeks); Stage 18, 44 days (approx. 6.3 weeks) and Stage 21, 51 days (approx. 7.3 625 weeks). s, subclavian artery; a, axillary artery; b, brachial artery; r, radial artery; u, ulnar artery; ai, anterior interosseous; $\mathrm{H}$, humerus; $\mathrm{U}$, ulna; $\mathrm{R}$, radius.

628 B Lower limb arterial transition. 12mm stage (approx. 5.4 weeks), 14mm stage (approx 5.7 629 weeks), $18 \mathrm{~mm}$ stage (approx. 6.3 days), $22 \mathrm{~mm}$ stage (approx. 6.8 weeks), Adult stage (after 7 th 630 week).

631 12mm stage of development a single-axis artery traverses the leg bud. By $14 \mathrm{~mm}$ two arteries (TP 632 and PPS) originate posteriorly and the RPC branches anteriorly off the Interossea artery. By $63318 \mathrm{~mm}$ the anterior tibial artery develops as a continuation of the RPC. A branch (the RC) 634 develops off the PPS and anastomoses with the interossea artery. Five arteries now traverse the 635 forming leg. At $22 \mathrm{~mm}$ stage of development there is regression of the distal part of the peronea 636 posterior superficialis and of the proximal part of the interossea artery. Note the presence of the 637 fibular (peroneal) artery (PE) which has formed from parts of the Axial, PPS, RC arteries. By the $6387^{\text {th }}$ week the adult pattern has emerged with the regression of the popliteal profunda.

639 Embryonic arteries: PP popliteal profunda; PS popliteal superficialis; PPS peronea posterior 640 superficialis; I interossea; RC ramus communicans inferior; RPC ramus perforans cruris; IS 641 ischiatic; TP tibialis posterior superficailis; P popliteal; PT posterior tibial; PE peroneal; AT 642 anterior tibial; RT recurrent tibial. 
Muscles: MP popliteus; MTP tibilis posterior; MFH flexor halluces.

644 Figure A reproduced with permission from M. Rodriguez-Niedenfuhr, G. J. Burton, J. Deu, J. R. 645 Sanudo, Development of the arterial pattern in the upper limb of staged human embryos: normal 646 development and anatomic variations. J. Anat. 199 (2001) 407-417. [13]

647 Figure B reproduced with permission from E. M. Levinsohn, D. R. Hootnick, D. S. Packard Jr. 648 Consistent arterial abnormalities associated with a variety of congenital malformations of the 649 human lower limb. Invest Radiol. 26 (1991) 364-373. [12]

\section{Figure 4}

\section{Altered/missing arterial patterns in legs with abnormalities}

653 Drawings of arterial patterns in A. Normal Limb; B. Limb with skeletal dysplasia with 654 abnormally short fibula - the anterior tibial and fibular (peroneal) arteries are absent and 655 functional embryonic arteries (I, PRS, RC) remain; C. Limb with skeletal dysplasia and absent 656 fibula with midline metatarsal dysplasia. Anterior tibial and fibular (peroneal) arteries are absent. 657 Functional embryonic arteries (I, RC) remain; D. Clubfoot, the anterior tibial artery is reduced. A 658 functional embryonic artery (PPS) is present.

659 These drawings are summary diagrams made following dissection of human tissues to analyse 660 the vessel pattern changes and the causation of the deformity [12, 17, 23, 33, 37].

661 Figure A-D from D. R. Hootnick, D. S. Packard Jr, E. M. Levinsohn, R. Constantine, 662 Postoperative necrosis in Clubfoot: recent findings and review. Orthopedics International Edition 663 1992; 1 (1): 48-68. Reprinted with permission from SLACK Incorporated [81] 


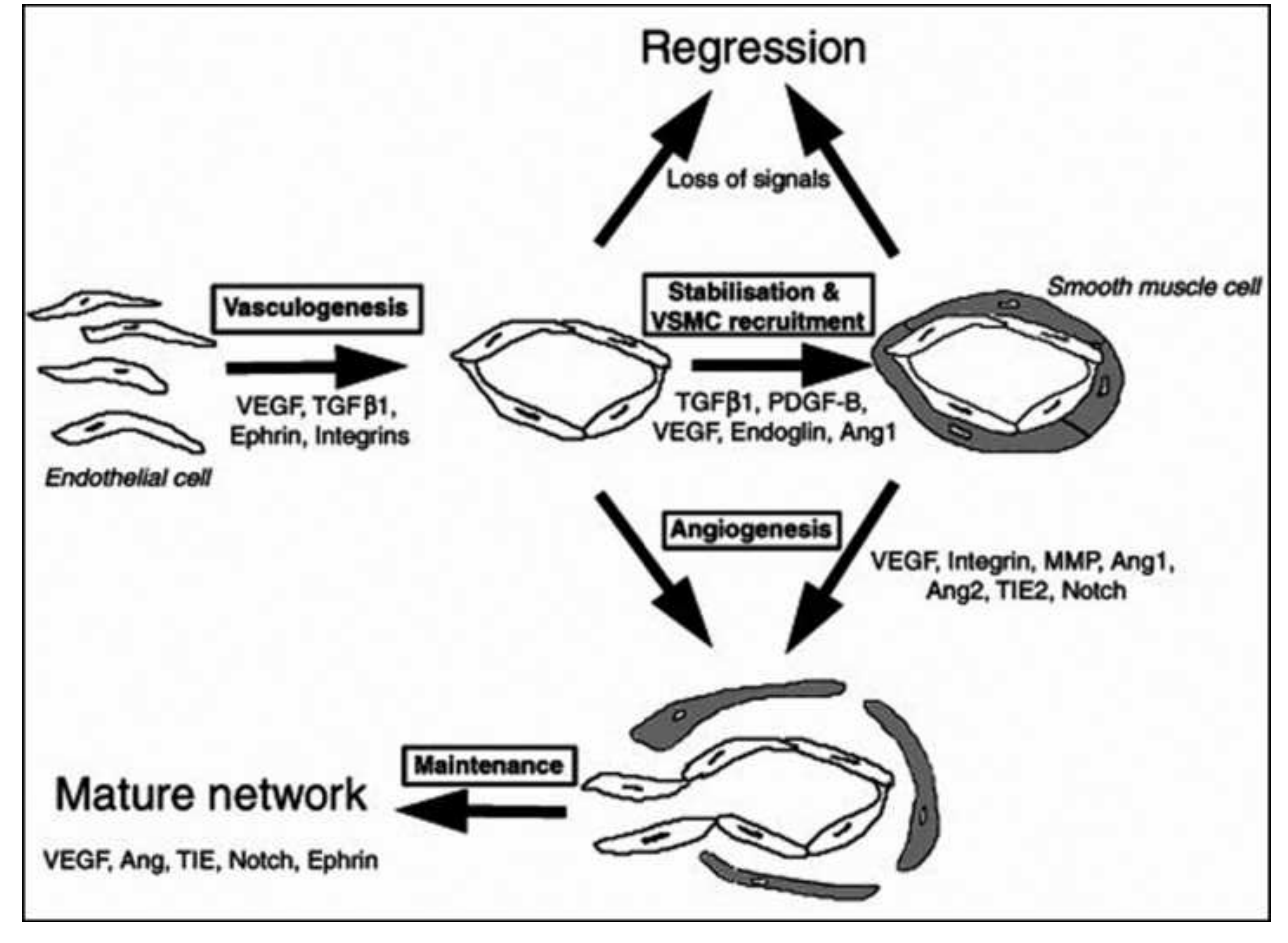

.

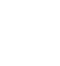


Click here to download high resolution image

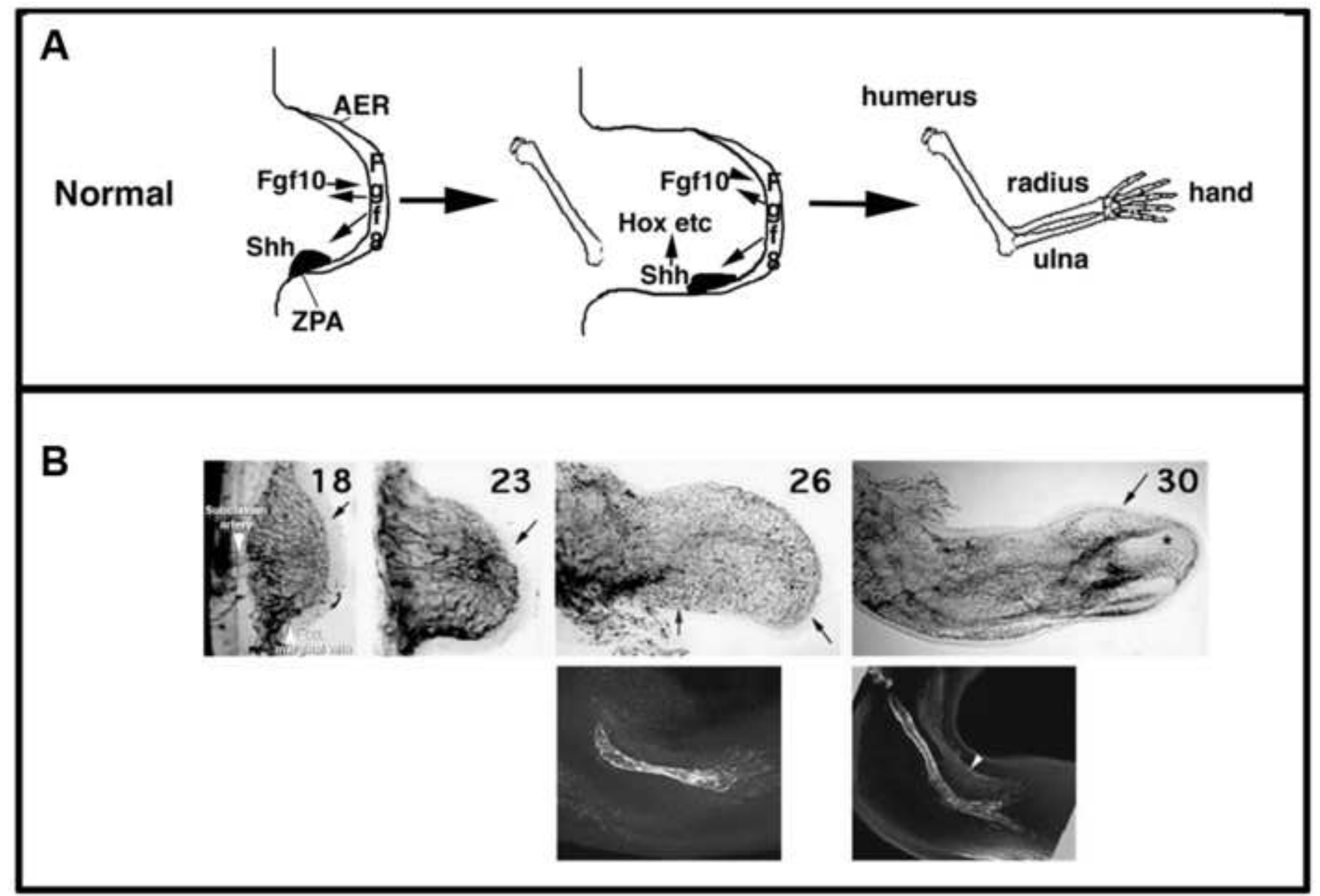


Click here to download high resolution image

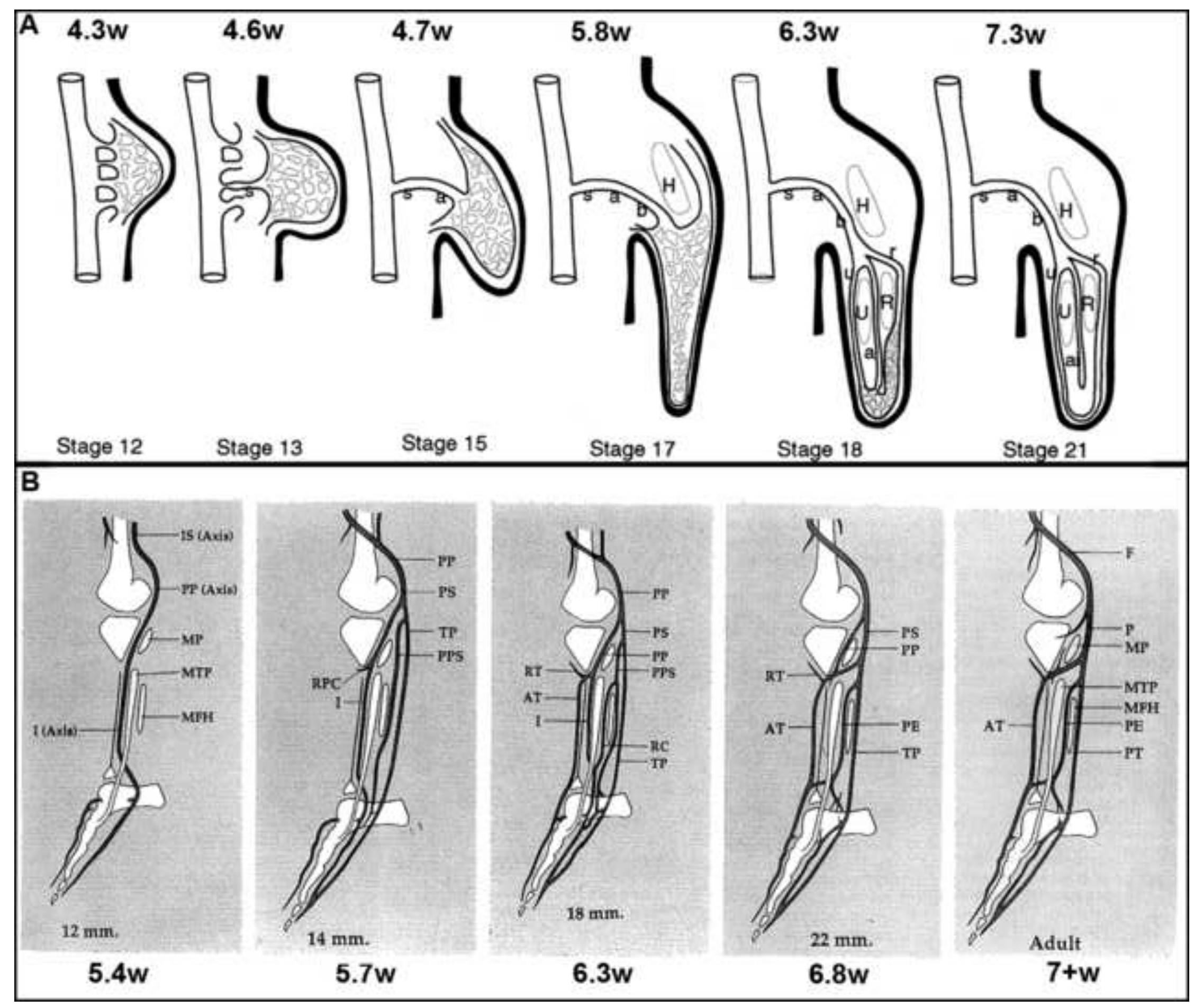


A

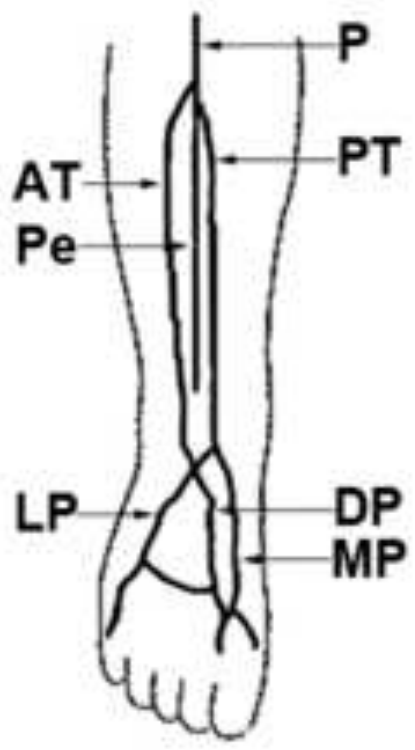

B

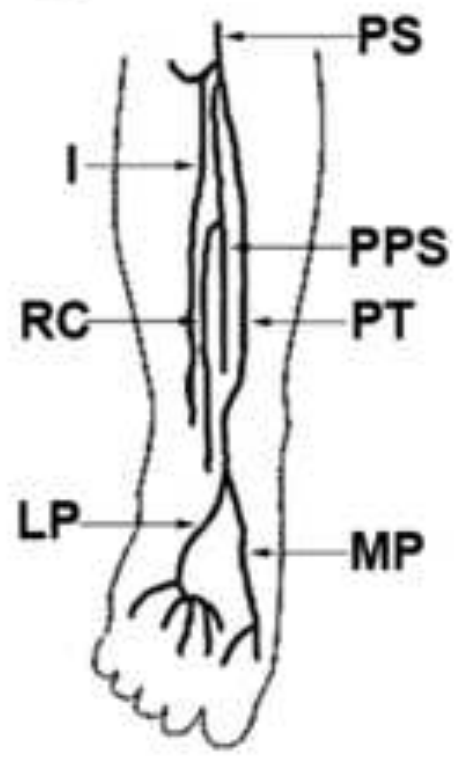

C

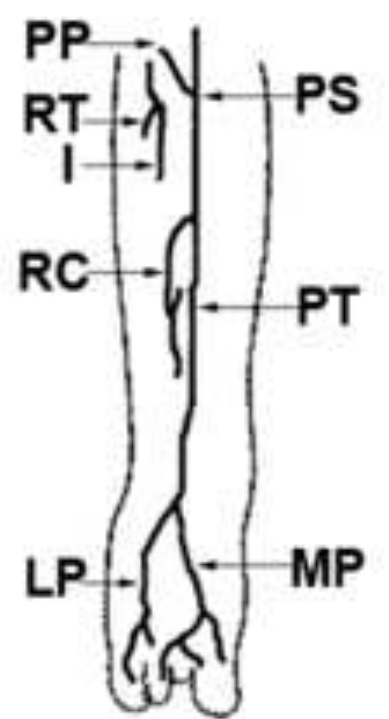

D

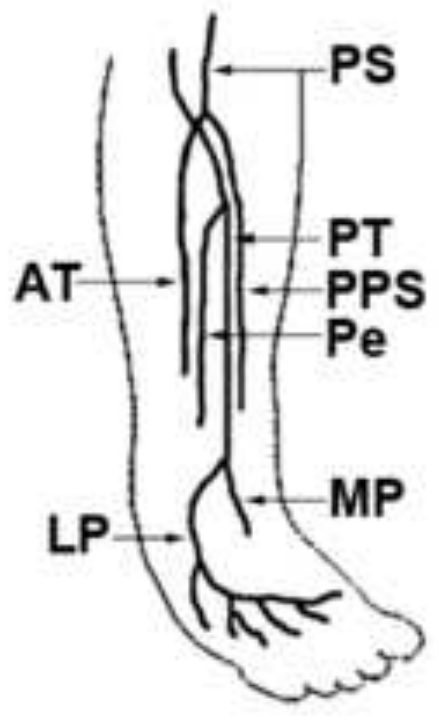

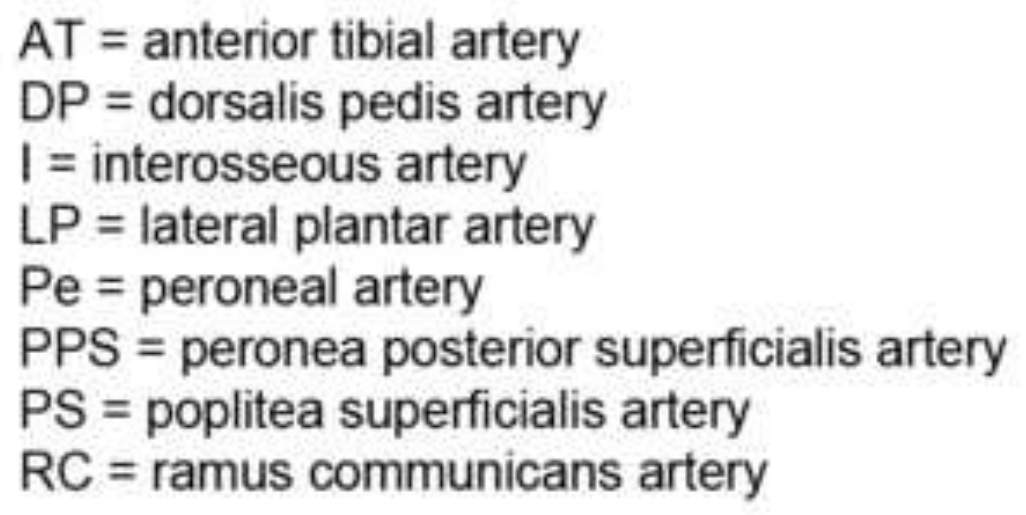


*Conflict of Interest Statement
Click here to download Conflict of Interest Statement: VARGESSON coi_disclosure.pdf

*Conflict of Interest Statement
Click here to download Conflict of Interest Statement: VARGESSON coi_disclosure.pdf

Click here to download Conflict of Interest Statement: VARGESSON coi_disclosure.pd 
*Conflict of Interest Statement
Click here to download Conflict of Interest Statement: HOOTNICK coi_disclosure.pdf

*Conflict of Interest Statement
Click here to download Conflict of Interest Statement: HOOTNICK coi_disclosure.pdf

Click here to download Conflict of Interest Statement: HOOTNICK coi_disclosure.pdf 